\title{
Location-based expert system for diabetes diagnosis and medication recommendation
}

\author{
Mohammed A. Almulla \\ Dept. of Computer Science, Kuwait University, Kuwait \\ Corresponding Author: almulla@cs.ku.edu.kw
}

\begin{abstract}
Using expert systems in the medical field has been practiced continuously for the past decades. There are attempts of using expert systems for a diabetes diagnosis. In this paper, we go further by proposing an expert system that not only diagnoses diabetes but also recommends the right medication depending on the location where the patient lives and on the symptoms of the patient and other effective factors. This system can be very helpful to many diabetic patients, especially to those who are not aware of their disease type or how to control it. The system outputs a list of names of locally available brand names of medications that suit the diabetes type of the patient and that do not pose any danger to the health of the patients according to their symptoms, effective factors, and results of the patients' medical tests. Our expert system is capable of reasoning using either forward chaining or backward chaining. The rules in the knowledge base are collected from several medical textbooks and articles published in scientific journals, periodicals, and international conferences. To verify the content of the knowledge base, a medical expert and a pharmacist working in Kuwait were consulted.
\end{abstract}

Keywords: Backward chaining; diabetes diagnosis; forward chaining; rule-based expert system; recommender systems.

\section{Introduction}

Diabetes is a progressive and chronic disease that threatens the lives of patients if not properly treated. Controlling this disease is considered a major health challenge, and its prevalence is increasing at an alarming rate (Holt et al., 2010). According to the World Health Organization, Diabetes Country Profiles 2016 on the prevalence rates of diabetes among Kuwait population and other related risk factors are as follows: diabetes $14.7 \%$, overweight $73.7 \%$, obesity $38.3 \%$, and physical inactivity $53.6 \%$ (Sminkey, 2016). The chairperson of Kuwait Diabetes Society Dr. Walid Al-Dhahi warned that diabetes is widely spread in Kuwait, as its prevalence rate has reached $24 \%$, and there are now more than 420,000 registered diabetes patients in Kuwait (Saleh, 2018). Diabetes is classified into the following four types: Type 1 (juvenile or insulindependent) may account for $5-10 \%$ of all diagnosed cases of diabetes; Type 2 (adult-onset or non-insulin-dependent) may account for $90-95 \%$ of all diagnosed cases of diabetes; prediabetes occurs when the amount of glucose in the blood is above the normal rate yet not high enough to be called diabetes; and gestational diabetes may account for $2-5 \%$ of all pregnancies but usually disappears when the pregnancy is over (Zeki et al., 2012; U.S. Department of Health and Human Services et al., 2012). Dr. Al-Dhahi asserted that $95 \%$ of the registered diabetes cases are of Type 2, adding the fact that obesity, high cholesterol levels, and blood pressure may accompany the disease. Moreover, Al-Adsani \& Abdula (2011) conducted a study to determine the prevalence and reasons for hospitalization of adults with diabetes in Kuwait of all hospital admissions in the Department of Medicine at Al-Sabah Hospital in Kuwait for 2 months. The results indicated that diabetes was the principal or secondary diagnosis in $40.6 \%$ of the hospitalizations.

The Ministry of Health $(\mathrm{MoH})$ is the main health care provider in Kuwait followed by some private hospitals and clinics distributed around the 6 municipalities of Kuwait. Dasman Diabetes Center, $\mathrm{MoH}$ through its public hospitals and clinics, and some private hospitals and clinics prescribe several medications for diabetes patients. These medications vary according to the patient's symptoms, signs, and lifestyle. If these symptoms are present in a patient, then the patient should refrain from taking this particular medication. Medical errors have killed so 
many patients in the past (Bao \& Jiang, 2016). Accurate diagnosis often depends on the physician's experience; however, many hospitals and clinics do not have medical experts for critical diseases such as diabetes. Moreover, prescribing wrong medications may cause a great physical and psychological burden for a patient on top of their high costs. Nonetheless, it may be hard to avoid mistakes. A study by Miller \& Mansingh (2017) indicated that, in a given year, more people die from medical errors than from car accidents, breast cancer, or AIDS. In this work, we propose a location-based medical expert system that checks the given signs and symptoms of a patient and makes some recommendations accordingly. Many types of recommendation techniques are found in the literature including Content Based (CB), Knowledge Based (KB), Collaborative Filtering (CF), and any hybrid of these techniques (Stark et al., 2019; Ricci et al., 2015). Our system uses the KB technique for diagnosing diabetes and recommending appropriate medications.

When designing an expert system, one of the primary goals of such an expert system is to have it solve problems as domain experts do. Furthermore, the typical structure of a general rule-based expert system requires the separation of the knowledge base from the inference engine. This separation makes it possible to use the same expert system's shell for building expert systems in various domains. However, this is not the case in our expert system. The main contribution of this work is to propose a location-based medical expert system for diabetes diagnosis and medication recommendation. Our system aims to provide flexible, yet accurate, and comprehensive recommendations. The brand names of the medications may vary according to the location where the patient lives. Hence, the system can recognize the type of diabetes based on the patient's signs, symptoms, and the results of the medical tests. The system's variables (i.e., patient's basic symptoms) for all types of diabetes are shown in Table 1. There are 115 different variables in this table. Accordingly, the system suggests medications that do not impose any danger on the medical condition of the patient, taking into consideration the patient's other diseases.

Table 1. Patient symptoms.

\begin{tabular}{lll}
\hline headaches & blood sugar level & irritability \\
increased thirst & slow healing wounds & rapid heartbeat \\
excessive thirst & darkened skin in armpits and neck & lethargy \\
itchy-skin & depression-and-stress & lightheadedness \\
dizziness & increased urge to urinate & excessive eating \\
dry skin & difficulty concentrating & excessive hunger \\
shakiness & irregular heartbeat & blurry vision \\
sweating & recurrent fungal infection & freq. infections \\
fatigue & recurring gum infection & thirst \\
fruity breath & excessive urination & nausea \\
pale skin & tingling sensation & weight loss \\
overweight & urinary tract infection & no increase thirst \\
high BP & no imp4aired vision & not overweight \\
high cholesterol & no increased appetite & weight variation \\
in urin ketones & no increased urge to urinate & weight reduction \\
hunger & hemoglobin variant & bad coordination \\
heart-ache hist. & polycystic ovary syndrome & convulsions \\
darkened skin & in blood autoantibodies & confusion \\
bed wetting & obesity & mood change \\
\hline & & \\
& &
\end{tabular}


In addition to the patient's symptoms, the user must enter the patient's signs (some call them the patient's demographic data) such as age, gender, waist size, bariatric surgery (if any), waist to hip ratio, family history of diabetes, pregnancy in case of female patients, previous pregnancies, and the patient's family history of diabetes during pregnancy.

The remainder of the paper is organized as follows. Section 2 discusses the related work. Section 3 describes the proposed expert system. Section 4 describes the knowledge base and the inference engine of the system. Section 5 provides the system evaluation and discussion. The last section contains the conclusion and future work.

\section{Related work}

Expert systems have been used in the medical field for so long. One can find many examples of medical expert systems such as MYCIN, MEDI, GUIDON, DIAGNOSER, and NURSExpert. When designing expert systems, the aim is to have them solve problems and/or make decisions as domain experts do. The above systems have been used to accurately diagnose various types of diseases such as microbial, bacterial, blood diseases, and depression. When it comes to diabetes diagnosis, the four reasoning methodologies that are commonly used in developing diabetic expert systems, namely, reasoning using production rules, fuzzy reasoning, case-based reasoning, and ontological case-based reasoning, were explored (Ahmed et al., 2015). The authors concluded that case-based reasoning is the best among the four methodologies for developing diabetic expert systems. Choubey et al. (2017) proposed an expert system that can diagnose diabetes at early stages with minimum time in an efficient manner. One year earlier, they proposed classification techniques for diagnosing diabetes (Choubey et al. 2016).

Dhivya \& Flix (2018) proposed a fuzzy rule-based expert system for Type 2 Diabetes Mellitus (T2DM) Diagnosis. Their system can make decisions and deal with ambiguous data with the aim of diagnosing diabetes at the early stages of the disease. Garcia et al. (2001) described ESDIABETES that was developed at Texas A\&M University-Corpus Christi to help people monitor and control the blood glucose level in their bodies. Humar \& Novruz (2008) presented a new method for classification of data in a medical database using a hybrid of a typical artificial neural network (ANN) and a fuzzy one. Margret et al. (2013) used a rough set system to develop a diabetes diagnosis system in which the first approximation sets are generated, and then the diagnosis is performed by considering those objects. Another expert system for diabetes diagnosis was proposed by Prajapati et al. (2016); this system was developed to assist the general practitioner in diagnosing and predicting the condition of a patient.

Recommender systems that are found in the literature aim to provide flexible, yet accurate, and comprehensive recommendations. To do so, they follow different types of recommendation techniques, each of which has its advantages and disadvantages. For example, Bao and Liang (2016) used the hybrid technique to suggest a design for an intelligent recommender system framework, whereas Miller \& Mansingh (2017) used two approaches, namely, Case-Base Reasoning (CBR) and Analytic Hierarchy Process (AHP) to design a distributed intelligent mobile agent decision-support system for optimal patient drug prescription. Another interesting attempt was proposed by Stark et al. (2017), where they used collaborative filtering and graph database technology to present a migraine drug recommender system based on Neo-4J. Their system gave recommendations with good accuracy.

Chen et al. (2011) used an ontology approach for antidiabetic drug recommendations to propose a recommender system based on multiple criteria decision-making and domain ontology. This system was meant to assist doctors to make more appropriate decisions about prescribing drugs via computed weights and ranks of these drugs. Medvedeva et al. (2007) suggested a case-similarity retrieval system in the form of a web-based application for assisting doctors to pool their knowledge to make confident decisions for the treatment of diabetes. Finally, Mahmoud \& Elbeh (2016) proposed an individualized Type 2 diabetes treatment through a drug recommender system that depends on ontology and Semantic Web Rule Language (SWRL).

\section{3. +Location-based expert system for diabetes diagnosis and recommendation}

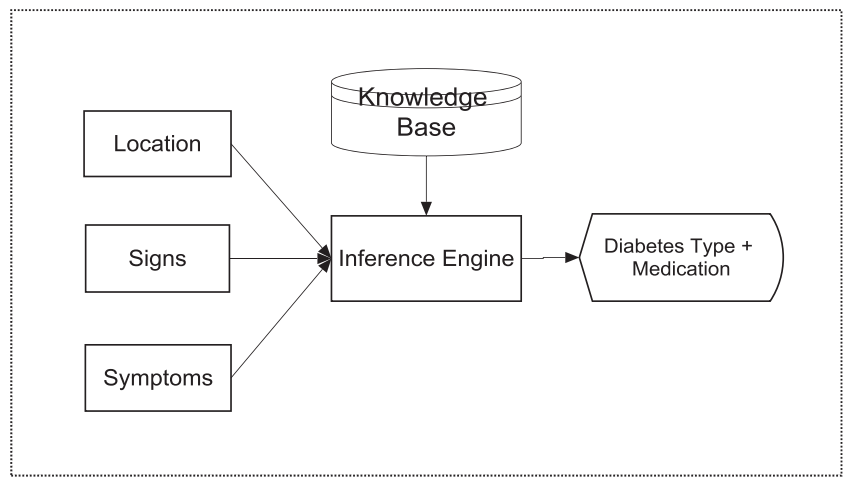

Fig. 1. Structure of the expert system. 
The proposed medical expert system can diagnose diabetes as well as recommend medications that can be taken by the patient without causing the patient any medical complications. Figure 1 shows the structure of the proposed system. In medicine, a contraindication of a prescribed medication is a condition or a factor that serves as a reason to withhold the medication due to the harm that it would cause to the patient (Vorvick, 2013). The knowledge base of our system contains rules that describe the contraindications of all medications the system is aware of, and hence the system does not recommend medicine if its contraindications conflict with the patient's symptoms. Moreover, each medicine has a generic name and a brand (commercial) name. For example, Metformin is the generic name of the medicine for Type 2 diabetes mellitus, and Fortamet, Glucophage XR, and Glumetza are its brand names (FDA Office of Women's Health, 2015). Some countries do not allow the import of some of these brand names due to different reasons. Hence, the system requires the location of the patient to suggest locally available brand names. Last but not least, the system is aware of the side-effects of all the medical treatments it recommends to the patient. Approximately, the knowledge base consists of 100 rules. The rules in the knowledge base are collected from textbooks (Agabegi \& Agabegi, 2020; Creutzfeldt, 1988; and U.S. Department of Health and Human Services et al. 2012), scientific journals (Choubey et al., 2017; Medvedeva et al., 2007; Mishra et al., 2016; and Stark et al., 2019), and international conferences (Hamed et al., 2012). Our expert system consists of 12,000 lines of code written in $\mathrm{C}$ programming language.

\section{The knowledge base and inference engine of the expert system}

The control strategy of the expert system presented in this work can be data-driven (forward chaining), where rules are applied whenever their left-hand-side conditions are satisfied, or goal-driven (backward chaining), where the system focuses its efforts by only considering rules that apply to some particular goal (Buchmann \& Duda, 1982), but not a hybrid of both. The choice of the control strategy is left to the user to decide, depending on many factors including the reason for using the system whether it is for knowledge acquisition or knowledge verification and explanation. Besides, it depends on the number of initial facts in the working memory vs the number of situations accepted as conclusions. Finally, it depends on the average number of choices in each direction, i.e., the number of rules that become eligible in each firing cycle.
4.1 Separating knowledge from control

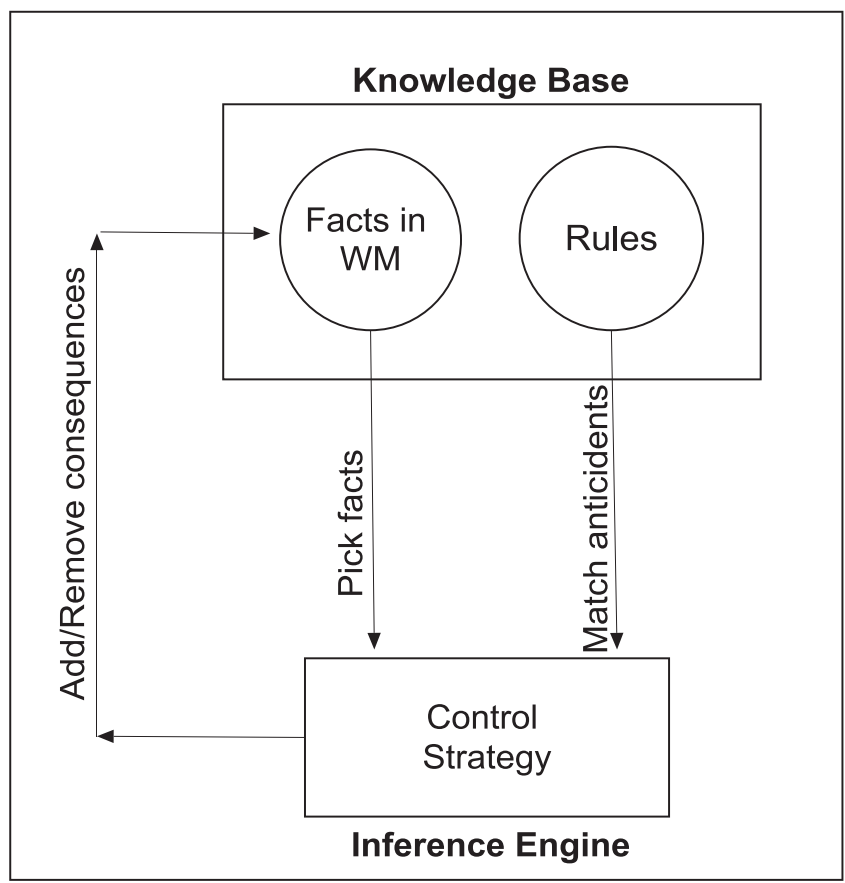

Fig. 2. Interaction between knowledge base and the inference engine.

One of the key principles of rule-based systems is the separation of knowledge (rules and working memory facts) from control (strategies of the inference engine). While control can be executed through forward or backward chaining, facts can be added to/removed from the working memory through the rules of the knowledge base. Note that, by using a backward chaining strategy, our rule-based system can justify, explain, and answer questions about its behavior.

Figure 2 shows the interaction between the knowledge base and the inference engine in the system. The knowledge base in the system consists of long-term memory, where the rules are stored, and the working memory, where the facts (assertions) are stored. A rule can have multiple antecedents joined by AND (conjunction), OR (disjunction), or a mixture of both. Our expert system can recognize only AND rules using the defrule function and OR rules using the deforule function. Our system can also handle mathematical operators to define numerical objects and assign numerical values to them. For instance, rule 57 says:

(defrule r57 (Type 2) (random_blood_sugar_test) (> blood_sugar_level 7.8) $(<=$ blood_sugar_level 11.1) $\Rightarrow$ (condition ?x prediabetes))

which means, for Type 2 patients, if patient X performed the random blood sugar test and the sugar level in the 
blood was between 7.8 and 11.1, then $\mathrm{X}$ has prediabetes condition. One unique characteristic of any expert system is its explanation facility. This feature enables the system to review its own reasoning and explain its decisions. The explanation facility in the system enables the user to ask the expert system how a particular conclusion was reached and why a certain fact was needed. Thus, the expert system can explain its reasoning and justify its advice, analysis, or decision. In the sequel, we demonstrate the sample runs for two simple problems: one that illustrates the use of an OR rule (deforule) and the second illustrates the contraindications of Metformin (medication for Type 2 diabetes patients).

Problem 1: The input to the system is the following:

\section{1}

2

(assert (A) (E))

(deforule r1 (A) $(\mathrm{B})=>(\mathrm{C}))$

(deforule $\mathrm{r} 2(\mathrm{X})(\mathrm{E})=>(\mathrm{Z}))$

(defrule $\mathrm{r} 3(\mathrm{C})(\mathrm{Z})=>(\mathrm{G})$ )

$($ deforule $($ test $(>15))($ test $(<15))=>(D))$

(run)

(facts)

The output of the system for Problem 1 is the following:

Select one of the following styles:

1-Forward Chaining

\section{2-Backward Chaining}

Your Choice must be between 1-2. Select a number: 1

Select one of the following strategies:

1-Stack (Last In First Out)

2-Queue (First In First Out)

3-Random Order

4-Hot List

5- Longest Matching

Your choice must be between 1 and 5. Select a number: 2

$>(\operatorname{assert}(\mathrm{A})(\mathrm{E}))$

$>($ deforule r1 $(\mathrm{A})(\mathrm{B})=>(\mathrm{C}))$
$>($ deforule $\mathrm{r} 2(\mathrm{X})(\mathrm{E})=>(\mathrm{Z}))$

$>$ (defrule $\mathrm{r} 3(\mathrm{C})(\mathrm{Z})=>(\mathrm{G}))$

$>($ deforule $\mathrm{r} 4($ test $(>15))($ test $(<15))=>(\mathrm{D}))$

$>$ (run)

Inference chain: r1-r2-r4-r3

$>$ (facts)

f0: (initial-fact)

f1: (A)

f2: (E)

f3: (C)

f4: $(Z)$

f5: (D)

f6: (G)

$>$

Problem 2: The input to the systems is the following: 1

1

(assert (location Kuwait) (Type unknown) (CHF) (not in_urin ketones) (blood_sugar_level 160) (sweating) (nausea) (hemoglobin_variant))

(defrule r1 (Type unknown) (not_in_urin ketones) $=>$ (Type 2))

(defrule r2 (Type 2) (sweating) (nausea) (hemoglobin variant $)=>$ (perform random_blood_sugar_test)

(defrule r3 (Type 2) (perform random_blood_sugar_test) $(>$ blood_sugar_level 140) $(<=$ blood_sugar_level 200) $=>$ (condition prediabetes $))$

(defrule $\mathrm{r} 4$ (Type 2$)=>($ medication metformin $))$

(defrule $\mathrm{r} 5$ (medication metformin) $=>$ (contraindication hypersensitivity_to_metforminCHFdiabetic_ketoacidosis renal_disease abnormal_creatinine_clearance lactation))

(defrule r6 (medication metformin) $=>$ (consumption metformin_oral))

(defrule $r 7$ (location Kuwait) (medication metformin) $=>$ (brand_name metaphage))

(defrule r8 (Type 2$)=>$ (medication sulfonylureas))

(defruler 9 (medicationsulfonylureas $)=>(($ contraindication sulfa_allergy Type_1_diabetes diabetic_ketoacidosis concomitant_use_with_bosentan)) 
(defrule r10 (medication sulfonylureas) $=>$ (action increase_beta-cell_insulin_secretion decrease_hepatic_ glucose_output increase_insulin_receptor_sensitivity))

(defrule r11 (location Kuwait) (medication sulfonylureas) $=>$ (brand name glimepiride)

(run)

(facts)

The output of the system for Problem 2 is the following:

Select one of the following styles:

1-Forward Chaining

2-Backward Chaining

Your choice must be between 1 and 2 . Select a number: 1

Select one of the following control strategies:

1-Stack (Last In First Out)

2-Queue (First In First Out)

3-Random Order

4-Hot List

5- Longest Matching

Your choice must be between 1 and 5. Select a number: 1 $>$ (assert (location Kuwait) (Type unknown) (CHF) (not_ in_urin ketones) (blood_sugar_level 160) (sweating) (nausea) (hemoglobin_variant))

$>$ (defrule r1 (Type unknown) (not_in_urin ketones) $=>$ (Type 2))

$>$ (defrule r2 (Type 2) (sweating) (nausea) (hemoglobin_ variant $)=>($ perform random_blood_sugar_test $))$

$>$ (defrule r3 (Type 2) (perform random_blood_sugar_ test) $(>$ blood_sugar_level 140) $(<=$ blood_sugar_level 200) $=>($ condition prediabetes $))$

$>($ defrule $\mathrm{r} 4$ (Type 2$)=>($ medication metformin $))$

$>($ defrule $r 5$ (medication metformin $)=>($ contraindication hypersensitivity_to_metforminCHFdiabetic_ketoacidosis renal_disease abnormal_creatinine_clearance lactation))

$>$ (defrule r6 (location Kuwait) (medication metformin)

$=>$ (brand_name metaphage)

$>($ defrule $r 7$ (Type 2$)=>($ medication sulfonylureas $))$

$>$ (defrule r8 (medication sulfonylureas) $\Rightarrow$ ((contraindication sulfa_allergy Type_1_diabetes diabetic_ketoacidosis concomitant_use_with_bosentan))
$>$ (defrule r9 (location Kuwait) (medication sulfonylureas)

$=>$ (brand_name glimepiride) $)$

$>$ (run)

Inference chain: r1-r2-r4-r7-r3-r6-r5-r9-r8

$>$ (facts)

f0: (initial-fact)

f1: (location Kuwait)

f2: (Type unknown)

f3: (CHF)

f4: (not_in_urin ketones)

f5: (blood_sugar_level 160)

f6: (sweating)

f7: (nausea)

f8: (hemoglobin_variant)

f9: (Type 2)

f10: (perform random_blood_sugar_test)

f11: (condition prediabetes)

f14: (medication sulfonylureas)

f15: (brand_name glimepiride)

$>$

It can be seen from the system that, after determining that the patient is diabetes of Type 2 , the two medications that can be suggested for this patient are Metformin and Sulfonylureas, but since this patient has CHF (symptom), then Metformin cannot be recommended and facts $\mathrm{f} 12$ and f13 were removed from the working memory. Hence, the system only suggested Sulfonylureas (the generic name) and glimepiride (the brand name).

\subsection{Learning module of the proposed system}

Assume that we have two rules in the knowledge base (KB), one that says $A=>B$ and the other that says $B$ $=>\mathrm{C}$, then the learning module enables the system to minimize the deduction process by introducing to the $\mathrm{KB}$ a new rule that says $\mathrm{A}=>\mathrm{C}$. Although this newly added rule will shorten the length of the deduction, it might take the system long time to reach the same conclusion, since there are more rules to be considered now. In our expert system, the learning module is a switch that is left to the user to decide whether to activate it or not. However, for our system, the speed of deciding to determine the 
diabetes type and medication treatment is very important. Even the most accurate diagnosis is useless if it arrives too late, for example, in an emergency case when the diabetes patient is about to enter in a comma stage. In the sequel, we illustrate a sample run for a simple problem that invokes OR rules.

Problem 3: The input to the systems is the following: 1 $(\operatorname{assert}(\mathrm{A})(\mathrm{Z})(\mathrm{V}))$ (defrule r1 $(\mathrm{A})=>(\mathrm{B}))$ (defrule r2 $(\mathrm{B})=>(\mathrm{C})$ ) (defrule r3 (C) $=>(\mathrm{D})$ ) (defrule $r 4(D)=>(E))$ $($ defrule $r 5(\mathrm{E})=>(\mathrm{F}))$ (defrule r6 $(\mathrm{F})=>(\mathrm{G})$ ) (defrule r7 (A) $=>(\mathrm{I})$ ) (defrule r8 $(\mathrm{H})=>(\mathrm{O}))$ (defrule r9 $(\mathrm{B})=>(\mathrm{J}))$ (defrule r10 (C) $=>(\mathrm{L})$ ) (defrule r11 (D) $=>(\mathrm{N})$ ) (defrule r12 $(\mathrm{P})=>(\mathrm{Q})$ ) (defrule r13 $(\mathrm{Q})=>(\mathrm{R}))$ (defrule r14 $(\mathrm{E})=>(\mathrm{W})$ ) (defrule r15 $(\mathrm{F})=>(\mathrm{X}))$ $($ defrule r16 $(\mathrm{I})=>(\mathrm{Y}))$ (defrule r17 $(\mathrm{J})=>(\mathrm{U}))$ (defrule r18 (I) $(\mathrm{J})=>(\mathrm{K}))$ (defrule r19 $(\mathrm{K})(\mathrm{L})=>(\mathrm{M}))$ $($ defrule r20 $(\mathrm{M})(\mathrm{N})=>(\mathrm{G}))$

(defrule r21 (W) $(\mathrm{X})(\mathrm{Z})(\mathrm{V})=>(\mathrm{T}))$

$($ defrule r22 $(\mathrm{T})(\mathrm{Y})(\mathrm{U})=>(\mathrm{G}))$

(learn_rules)

(learned_rules)

(run)

(facts) (rules)

(print_stats)

The output of the system for Problem 3 is the following:

Select one of the following styles:

1-Forward Chaining

2-Backward Chaining

Your choice must be between 1 and 2. Select a number: 1

Select one of the following control strategies:

1-Stack (Last In First Out)

2-Queue (First In First Out)

3-Random Order

4-Hot List

5-Longest Matching

Your choice must be between 1 and 5. Select a number: 1

$>(\operatorname{assert}(\mathrm{A})(\mathrm{Z})(\mathrm{V}))$

$>($ defrule $\mathrm{r} 1(\mathrm{~A})=>(\mathrm{B}))$

$>($ defrule $\mathrm{r} 2(\mathrm{~B})=>(\mathrm{C}))$

$>($ defrule $r 3(\mathrm{C})=>(\mathrm{D}))$

$>($ defrule $\mathrm{r} 4(\mathrm{D})=>(\mathrm{E}))$

$>($ defrule r5 $(\mathrm{E})=>(\mathrm{F}))$

$>($ defrule r6 $(\mathrm{F})=>(\mathrm{G}))$

$>($ defrule $\mathrm{r} 7(\mathrm{~A})=>(\mathrm{I}))$

$>($ defrule $\mathrm{r} 8(\mathrm{H})=>(\mathrm{O}))$

$>($ defrule $\mathrm{r} 9(\mathrm{~B})=>(\mathrm{J}))$

$>($ defrule $\mathrm{r} 10(\mathrm{C})=>(\mathrm{L}))$

$>($ defrule r11 $(\mathrm{D})=>(\mathrm{N}))$

$>($ defrule $r 12(\mathrm{P})=>(\mathrm{Q}))$

$>($ defrule $r 13(\mathrm{Q})=>(\mathrm{R}))$

$>($ defrule r14 $(\mathrm{E})=>(\mathrm{W}))$

$>($ defrule $\mathrm{r} 15(\mathrm{~F})=>(\mathrm{X}))$

$>($ defrule $\mathrm{r} 16(\mathrm{I})=>(\mathrm{Y}))$

$>($ defrule $\mathrm{r} 17(\mathrm{~J})=>(\mathrm{U}))$

$>($ defrule r18 $(\mathrm{I})(\mathrm{J})=>(\mathrm{K}))$

$>($ defrule r19 $(\mathrm{K})(\mathrm{L})=>(\mathrm{M}))$ 
$>($ defrule $r 20(\mathrm{M})(\mathrm{N})=>(\mathrm{G}))$

$>($ defrule $\mathrm{r} 21(\mathrm{~W})(\mathrm{X})(\mathrm{Z})(\mathrm{V})=>(\mathrm{T}))$

$>($ defrule $\mathrm{r} 22(\mathrm{~T})(\mathrm{Y})(\mathrm{U})=>(\mathrm{G}))$

$>$ (learn_rules)

$>$ (run)

$>$ (facts)

f0: (initial-fact)

f1: (A)

f2: (Z)

f3: (V)

f4: (I)

f5: (Y)

f6: (B)

f7: (C)

f8: (L)

f9: (J)

f10: $(\mathrm{K})$

f11: (M)

f12: (U)

f13: (D)

f14: (E)

f15: (W)

f16: (N)

f17: (G)

f18: (F)

f19: (X)

f20: (T)

$>$ (rules)

Rules (* means already fired):

$\mathrm{r} 1: *($ Salience $=0)(\mathrm{A})==>(\mathrm{B})$

$\mathrm{r} 2: *($ Salience $=0)(\mathrm{B})==>(\mathrm{C})$

$\mathrm{r} 3 \mathrm{*}^{*}($ Salience $=0)(\mathrm{C})==>(\mathrm{D})$

$\mathrm{r} 4:^{*}($ Salience $=0)(\mathrm{D})==>(\mathrm{E})$

$\mathrm{r} 5 \mathrm{*}^{*}($ Salience $=0)(\mathrm{E})==>(\mathrm{F})$

r6:* $($ Salience $=0)(F)==>(G)$ r7:* $($ Salience $=0)(A)==>(I)$

r8: $($ Salience $=0)(\mathrm{H})==>(\mathrm{O})$

r9:* $($ Salience $=0)(\mathrm{B})==>(\mathrm{J})$

r10:* $($ Salience $=0)(\mathrm{C})==>(\mathrm{L})$

r11:* $($ Salience $=0)(\mathrm{D})==>(\mathrm{N})$

r12: $($ Salience $=0)(\mathrm{P})==>(\mathrm{Q})$

r13: $($ Salience $=0)(\mathrm{Q})==>(\mathrm{R})$

r14:* $($ Salience $=0)(\mathrm{E})==>(\mathrm{W})$

r15:* (Salience $=0)(\mathrm{F})==>(\mathrm{X})$

r16:* $($ Salience $=0)(\mathrm{I})==>(\mathrm{Y})$

r17:* $($ Salience $=0)(J)==>(U)$

r18:* $($ Salience $=0)(\mathrm{I})(\mathrm{J})==>(\mathrm{K})$

r19:* $($ Salience $=0)(\mathrm{K})(\mathrm{L})==>(\mathrm{M})$

r20:* $($ Salience $=0)(\mathrm{M})(\mathrm{N})==>(\mathrm{G})$

r21:* $($ Salience $=0)(\mathrm{W})(\mathrm{X})(\mathrm{Z})(\mathrm{V})==>(\mathrm{T})$

r22:* $($ Salience $=0)(\mathrm{T})(\mathrm{Y})(\mathrm{U})==>(\mathrm{G})$

r23: $($ Salience $=0)(\mathrm{A})==>(\mathrm{C})$

r24: $($ Salience $=0)(A)==>(J)$

r25: $($ Salience $=0)(B)==>(D)$

r26: $($ Salience $=0)(B)==>(L)$

r27: $($ Salience $=0)(C)==>(E)$

r28: $($ Salience $=0)(\mathrm{C})==>(\mathrm{N})$

r29: $($ Salience $=0)(D)==>(F)$

r30: $($ Salience $=0)(D)==>(W)$

r31: $($ Salience $=0)(E)==>(G)$

r32: $($ Salience $=0)(E)==>(X)$

r33: $($ Salience $=0)(\mathrm{A})==>(\mathrm{Y})$

r34: $($ Salience $=0)(B)==>(U)$

r35: $($ Salience $=0)(\mathrm{P})==>(\mathrm{R})$

r36: $($ Salience $=0)(A)==>(E)$

r37: $($ Salience $=0)(A)==>(N)$

r38: $($ Salience $=0)(B)==>(F)$

r39: $($ Salience $=0)(B)==>(W)$

r40: $($ Salience $=0)(\mathrm{C})==>(G)$

r41: $($ Salience $=0)(C)==>(X)$ 
$>$ (print_stats)

Number of facts in the WM= 21

Number of functions defined $=0$

Number of rules in the $\mathrm{KB}=41$

Number of rules fired $=17$

Number of templates defined $=0$

Number of global variables $=0$

Number of local variables $=0$

Inference chain: r7-r16-r1-r2-r10-r9-r18-r19-r17-r3-r4r14-r11-r20-r5-r6-r15-r21-r22

$>$

Matching the antecedents of the rules with the facts in the Working Memory is called the inference chain. This chain indicates how the expert system applies the rules to conclude. In addition to the inference chain, the above sample run shows the statistics produced by the system on the given problem. With the activation of the learning module, the total number of defined rules is 22 , and the total number of learned rules is equal to $41-22=19$ rules. Note that the fired rules are identified by an '*' printed after the rule name.

\section{Evaluation and discussion}

To evaluate the performance of the proposed system, we used an online dataset "diabetic_data.csv" that contains 101766 records of diabetic patients. These data include patient demographics, history of health problems, current symptoms, pregnancy (if applicable), and other related factors such as accessibility of the medication (i.e., over-the-counter or needs doctor prescription). Among these data, we randomly selected 1000 patients' records. The dataset distinguished between 23 medications for diabetes, whereas our expert system can recognize 51 different medications for diabetes. We collected data about these 51 common medications for diabetes and their contraindications, effect, side-effects, and usage that may be recommended by the system and that are available in both Kuwait and USA. The patient dataset can be obtained online from the following website: https://www.kaggle. com/smit1212/diabetic-data-cleaning

In general, the objective of a generic recommendation system is to provide the user with a list of objects to choose from based on the user's individual features (Stark et al., 2017). For each of the randomly selected 1000 data records, we compared the diabetic type diagnosed by our system and the recommended list of medications with those reported in the dataset. The proposed system scored $\% 96$ success rate. In other words, the medication listed in the dataset was among the list of medications recommended by our system in 964 cases out of the 1000 data records. For the remaining 36 cases, either the patient has not been prescribed any medication according to the dataset or our expert system did not recognize the medication reported in the dataset.

The results of the proposed rule-based expert system indicated the retrieval accuracy of $100 \%$ with correct symptoms. Hence, the evaluation shows that the proposed system is very promising. Not only the type of diabetes was correctly diagnosed, but also the medications recommended by the system were suitable to the patient's condition based on the patient demographics, history, symptoms, and other related factors.

\section{Conclusion and future work}

This research confirms the effective role of expert systems in the medical science and health care field. The proposed expert system in this paper not only can predict the presence of diabetes in the patient at the early stages, but also can suggest medication that suits the patient's medical condition taking into account the patient's symptoms and demographic data. The system can easily be extended to recognize further brand names prescribed in other locations. However, the patients are advised to consult their diabetes doctors before taking the recommended medications.

One should realize that expert systems' knowledge is based on the knowledge of the experts, and hence these systems are designed to perform at the human expert level. These experts are only human, and their decisions might not always be accurate, no matter how careful they are. Consequently, expert systems should be allowed to make mistakes just like the domain experts do. Nonetheless, we still trust the experts even though we know their decisions are not always accurate. Likewise, we can accept the decisions made by expert systems.

Current limitations of the system are as follows: (1) the system can be a reason beyond its domain of discourse (i.e., the system is unable to reason outside its area of expertise). (2) The system is unable to reason with incomplete or uncertain knowledge. In the future, we plan to use this system for other widely spread diseases such as headaches/migraines and heart attacks. In addition, we plan to add certainty factors to the rules of the KB for reasoning 
with uncertainty. Finally, more efficient implementations of the firing order of the rules are possible. We plan to apply heuristics to guide the reasoning and thus reduce the search for a solution.

\section{ACKNOWLEDGEMENTS}

The author would like to acknowledge and sincerely thank all those inspired, supported, and helped in the process of collecting and verifying the knowledge before storing it in the knowledge base of the expert system.

\section{References}

Agabegi, S. \& Agabegi E. (2020). STEP-UP to Medicine, $5^{\text {th }}$ Edition, Wolters Kluwer Publishers, China.

Ahmad, I.M., Alfonse, M., Aref, M. \& Salem, A.M. (2015). Reasoning Techniques for Diabetics Expert Systems. Science Direct: Procedia Computer Science, 65: 813-820.

Al-Adsani, A. \& Abdulla, Kh. (2011). Reasons for hospitalizations in adults with diabetes in Kuwait. International Journal of Diabetes Mellitus, 3(1): 65-69.

Bao, Y. \& Jiang, X. (2016). An intelligent medicine recommender system framework. IEEE $11^{\text {th }}$ Conference on Industrial Electronics and Applications (ICIEA), pp. 1383-1388.

Buchmann B. \& Duda, R. (1982). Principles of RuleBased Expert Systems. Advances in Computers, 22:163216.

Chen, R.C., Chiu, J.Y. \& Bau, C.T. (2011). The Recommendation of Medicines Based on Multiple Criteria Decision Making and Domain Ontology- An Example of Anti-Diabetic Medicines. Proceedings of the International Conference on Machine Learning and Cybernetics, Guilin, 10-13 July 2011.

Choubey, D.K., Paul, S. \& Dhandhenia, V.K. (2017). Rule-based diagnosis system for diabetes. Biomedical Research, 28(12): 5196-5209.

Choubey, D.K. \& Paul, S. (2016). Classification techniques for the diagnosis of diabetes: a review. International Journal of Biomedical Engineering and Technology, 21(1): 15-39.

Creutzfeldt, W. (1988). Acarbose for the Treatment of Diabetes Mellitus, Conference Proceedings, SpringerVerlag.

De Tore, A. W. (1989). An Introduction to Expert
Systems. Journal of Insurance Medicine, 21(4): 233-236.

Dhivya, A.D. \& Felix, A. (2018). A Fuzzy Rule-based Expert System for T2DM Diagnosis. International Journal of Engineering \& Technology, 7(4): 432-435.

FDA Office of Women's Health. (2015). Diabetes Medicines. Retrieved on 2 Feb. 2020, https://www.fda. gov/media/91130/download

Garcia, M., Gandhi, A., Singh, T., Duarte, L., Shen, R., Dantu, M., Ponder, S. \& Ramirez, H. (2001). Esdiabetes (an expert system in diabetes). Journal of Computing Sciences in Colleges, 16(3): 166-175.

Hamed, A.A., Roose, R., Branicki, M. \& Rubin, A. (2012). T-Recs: Time-aware Twitter-based Drug Recommender System. IEEE/ACM International Conference on Advances in Social Networks Analysis and Mining, pp. 1028-1031.

Holt, R.I., Cockram, C.S., Flyvbjerg, A. \& Goldstein, B.J. (2010). Textbook of Diabetes. $4^{\text {th }}$ Edition, WileyBlackwell Publishers.

Humar, K. \& Novruz, A. (2008). Design of a hybrid system for diabetes and heart diseases. Expert Systems \& Applications, Elsevier, 35: 82-89.

Mahmoud, N. \& Elbeh, H. (2016). IRS-T2D: Individualize Recommendation System for Type2 Diabetes Medication Based on Ontology and SWRL. Proceedings of the $10^{\text {th }}$ International Conference on Informatics and Systems, May 2016, pp. 203-209.

Margret, A.S., Clara, M.L., Jeevitha, P. \& Nandhini, R.T. (2013). Design of a Diabetic Diagnosis System Using Rough Sets. Cybernetics and Information Technologies, 13(3): 124-139.

Medvedeva, O., Knox, T.L. \& Paul, J. (2007). DiaTrack: Web-Based Application for Assisted Decision-Making in Treatment of Diabetes. Journal of Computing Sciences in Colleges. 23(1): 154-161.

Miller, K. \& Mansingh, G. (2017). Optipres: a distributed mobile agent decision support system for optimal patient drug prescription. Information Systems Frontiers, 19(1): 129-148.

Mishra, D., Painuli, D. \& Nirvikar, (2016). Rule Based Expert System for Medical Diagnosis-A Review. International Journal of Engineering Technology, Management and Applied Sciences, 4(12): 167-172.

Prajapati, H., Pal, S.K. \& Jain, A. (2016). Expert System for Diagnosis of Diabetes: A Review. International 
Journal of Advances in Engineering \& Technology, 9(5): 532-537.

Ricci, F., Rokach, L. \& Shapira, B. (2015). Recommender systems: introduction and challenges. Recommender Systems Handbook. Springer-Verlag, pp. 1-34.

Saleh, A. (2018). 420,000 diabetic patients in Kuwait. Kuwait Times, Retrieved on 12 Dec. 2018, https://news. kuwaittimes.net/website/420000-diabetic-patients-inkuwait/

Sharaa, A. (2019). 23\% Prevalence of Diabetes in Kuwait. Kuwait Times, Retrieved on 11/9/2019, https://news. kuwaittimes.net/website/23-prevalence-of-diabetes-inkuwait-doctor

Sminkey, L. (2016). Kuwait Profile: Diabetes Country Profiles 2016. World Health Organization, http://origin. who.int/diabetes/country-profiles/kwt_en.pdf

Stark, B., Knahl, C., Aydin, M. \& Elish, K. (2019). A Literature Review on Medicine Recommender Systems. International Journal of Advanced Computer Science and Applications (IJACSA), 10(8): 6-13.

Stark, B., Knahl, C., Aydin, M., Samarah, M. \& Elish, K. (2017). BetterChoice: A Migraine Drug Recommendation System Based on Neo-4J. In the $2^{\text {nd }}$ IEEE International Conference on Computational Intelligence and Applications (ICCIA), pp. 382-386.

U.S. Department of Health and Human Services, National Institutes of Health, and National Institute of Diabetes and Digestive, and Kidney Diseases, (2012). Your Guide to Diabetes: Type 1 and Type 2, CreateSpace Independent Publishing Platform, USA.

Vorvick, L.J., Zieve, D., Eltz, D.R., Slon, S. \& Wang, N. (2019). Contraindication: MedlinePlus Medical Encyclopedia. MEDLINE, United States National Library of Medicine, Retrieved on 6 Nov. 2019, https:// medlineplus.gov/ency/article/002314.htm

Zeki, T., Malakooti, M., Ataeipoor, Y. \& Tabibi, T. (2012). An Expert System for Diabetes Diagnosis. American Academic \& Scholarly Research Journal, 4(5): $1-13$.

Submitted : 25/11/2019

Revised : 04/02/2020

Accepted : 05/02/2020 